\title{
ORIGINAL
}

\section{CRIBAJE DE MICROFILARIASIS SANGUÍNEA (LOA LOA) EN LA POBLACIÓN INMIGRANTE DE ZONAS ENDEMICAS}

Esther Carrillo Casas (1), Begoña Iglesias Pérez (2), Jordi Gómez i Prat (3), Caterina Guinovart Florensa (4) y Juan Cabezos Otón (3)

(1) CAP St. Cugat del Vallès, H.Mutua de Terrassa. Barcelona.

(2) SAP Ciutat Vella. Barcelona.

(3) Unitat de Medicina Tropical i Salut Internacional Drassanes. SAP Ciutat Vella/Institut Català de la Salut. Barcelona.

(4) UASP, Hospital Clínic. Barcelona

\section{RESUMEN}

Fundamento: Dada la creciente afluencia de población inmigrante originaria de zonas de riesgo a nuestro país se plantea la necesidad de realizar cribaje para loasis, aunque el cribaje sistemático es una práctica discutida, poco evaluada y aún no protocolizada. E objetivo de este estudio es identificar la población en la que es más .

Métodos: Estudio de casos y controles, con 30 casos (todos los que consultaron en nuestra Unidad y con un test de detección de microfilaremia positivo para Loa loa) y 90 controles (tres controles por caso de entre los sujetos con microfilaremia negativa, escogidos aleatoriamente y sin criterios de apareamiento).

Resultados: De los 1.638 sujetos a los que se realizó la prueba de microfilaremia $30(1,8 \% ; 1,2-2,6 \%)$ resultaron positivos. De estos 30 casos con loasis $76,7 \%(23 \cdot 57,7-90,1 \%)$ presentaban eosinofilia (OR $8,8 ; 3,3-23,1 ; \mathrm{p}<0,0001)$ y un $30,0 \%(9 ; 14,7-49,4 \%)$ presentaba clínica (OR 2,8;1,0-7,5; $\mathrm{p}=0,04)$. Si aplicáramos el test de cribado nica $(\mathrm{OR} 2,8 ; 1,0-7,5 ; \mathrm{p}=0,04)$. Si aplicáramos el test de cribado a
toda la población inmigrante procedente de áreas endémicas deberíamos realizar 54,6 pruebas para detectar un caso. Si lo realizáramos en sujetos que presentan eosinofilia y/o clínica compatible, el número de pruebas que deberíamos realizar para cada caso detectado sería inferior $(\mathrm{NNS}=29 ; \mathrm{IC}=21-48)$ pero habría un $16,7 \%(5 ; 5,7-34,7 \%)$ de falsos negativos.

Conclusiones: Estaría indicado practicar un test de cribado con determinación de microfilaremia a la población inmigrante procedente de Africa Central y Oeste, independientemente de la pres (1) clínica compatible, siempre que se disponga de los recursos necesarios.

Palabras clave: Filariasis. Loa loa. Tamizaje. Inmigración.

Correspondencia:

Jordi Gómez i Prat

Unitat de Medicina Tropical i Salut Internacional Drassanes.

Av. de les Drassanes 17-21

08001 Barcelona

Correo electrónico: jordigp.pbcn@ics.scs.es
ABSTRACT

\section{Screening of Microfilariasis in Blood \\ (Loa Loa) among the Immigrant Population in Endemic Areas}

Background: Given the increasing flux of immigrant population from high-risk areas to our country, the need of screening for loasis arises, despite systematic screening being a debated and poorly evaluated practice to wich there is no protocol. This study is aimed at identifying the population to which loasis screening would be most appropriate, by drawing a comparison among four alternatives.

Methods: Case and control group study, involving 30 cases (all those who came to our unit for treatment and who tested positive for Loa Loa in a microfilariae in the blood detection test) and 90 control cases (three controls per case from among the subjects having tested negative for microfilariae in the blood chosen at random without any pairing criteria).

Results: Of the 1,638 subjects on whom the microfilariae blood test was performed, 30 tested positive $(1.8 \% ; 1.2-2.6 \%)$. Of these 30 cases of loasis, $76.7 \%(23 ; 57.7-90.1 \%)$ had eosinophylia (OR 8.8 $3.3-23.1 ; \mathrm{p}<0.0001)$ and $30.0 \%(9 ; 14.7-49.4 \%)$ compatible clinical symptoms (OR $2.8 ; 1.0-7.5 ; \mathrm{p}=0.04$ ). If we were to apply the screening test to the entire immigrant population coming from endemic areas, we would have to perform 54.6 tests to detect one case. It we were to perform the test on patients showing eosinophylia and/or compatible clinical symptoms, we would have to perform a smaller number of tests for every case detected (NNS=29; IC $=21-48)$, but there would be $16.7 \%(5 ; 5.7-34.7 \%)$ false negatives

Conclusions: Conducting a screening test with determination of microfilariae in the blood on the immigrant population coming from Central and West Africa, independently of the presence of eosinophylia or compatible clinical symptoms, would be indicated, provided that the necessary resources are available.

Key words: Filariasis. Loa loa. Screening. Immigration. 


\section{INTRODUCCIÓN}

La filariasis por el nemátodo Loa loa es una enfermedad infecciosa crónica, transmitida por una mosca del género Chrysops, endémica en la mayor parte de zonas forestales de países de África central y del oeste. Hasta 13 millones de personas pueden estar afectadas por la enfermedad en zonas hiperendémicas ${ }^{1}$, donde las tasas de infestación de la población autóctona y de los extranjeros residentes en estos países (expatriados) pueden llegar a ser del $100 \%$ y $30 \%$ respectivamente ${ }^{2,3}$.

Se trata de una enfermedad que puede pasar desapercibida por la escasez de síntomas en población nativa y, aunque en raras ocasiones, puede presentar complicaciones graves renales, cardíacas o de sistema nervioso central por fenómenos de hipersensibilidad $^{4,-6}$. La atención de estas complicaciones requiere el consumo de recursos sanitarios y una disminución de la calidad de vida de los sujetos. El comportamiento de Loa loa en el huésped definitivo está relacionado con el tiempo de exposición, de manera que las características clínicas y de laboratorio de la loasis en enfermos expatriados y nativos de áreas endémicas son distintas, debiéndose este fenómeno a las diferencias en la respuesta inmunológica desencadenada por la presencia de antígenos filáricos. En la población africana nativa de áreas endémicas, con una menor respuesta inmunitaria específica pero mayores niveles de microfilaremia en sangre, llama la atención la menor presencia de hipereosinofilia y de expresividad clínica de la enfermedad, en cambio los sujetos expatriados presentan más manifestaciones clínicas en respuesta a una hiperreactividad alérgica, más eosinofília y mayores elevaciones de inmunoglobinas ${ }^{7,8}$.

En sujetos expatriados procedentes de áreas endémicas para loasis el método más adecuado para realizar el cribaje general sería el contaje de eosinófilos ${ }^{9}$. En cambio, la determinación de microfilaremia se considera la técnica más indicada para el cribaje de población nativa a riesgo, procedente de África central y del oeste, siendo la sensibilidad de este método de entre un 75 y $90 \%$ en la población africana ${ }^{7,8,10,11}$.

El diagnóstico de loasis se realiza clásicamente mediante detección de microfilarias en sangre periférica, ya sea por extensión simple o con técnicas de concentración que aumentan la sensibilidad. Existen técnicas diagnósticas más modernas, sensibles y específicas pero de mayor coste económico basadas en serología (EIA y ELISA) o técnicas de detección de DNA y reacción en cadena de la polimerasa $(\mathrm{PCR})^{12-14}$, pero por su elevado coste no están recomendadas como pruebas de cribado.

Dada la creciente afluencia de población inmigrante originaria de zonas de riesgo a nuestro país se plantea la necesidad de realizar cribaje para loasis, aunque el cribaje sistemático es una práctica discutida, poco evaluada y aún no protocolizada en Catalu$\tilde{n} a^{9,11,15,16}$. Evaluar en qué población es más indicado realizar este tipo de cribado nos permitiría rediseñar los protocolos de acogida, mejorando así su uso.

En este estudio nos proponemos, por una parte, determinar la prevalencia de loasis en la población atendida en nuestra Unidad procedente de áreas endémicas e identificar factores de riesgo sociodemográficos, clínicos y de laboratorio para loasis en la población de estudio. Por otra parte, queremos identificar la población en la que es más adecuado realizar el cribado de loasis, comparando 4 alternativas: 1. determinación de microfilaremia sanguínea a toda la población procedente de áreas endémicas; 2. sólo a los sujetos que presentan clínica; 3. sólo a los que presentan eosinofilia periférica y 4. a los que presentan clínica y/o eosinofilia.

\section{SUJETOS Y MÉTODO}

El estudio se realizó en la Unidad de Medicina Tropical y Salud Internacional de 
Drassanes de Barcelona (España), centro del Institut Català de la Salut (ICS). A todos los sujetos que llegan a la Unidad procedentes de zonas endémicas para loasis (países de Africa central y del oeste) se les aplica sistemáticamente un protocolo de cribado, que incluye detección de microfilaremia.

En el período de tiempo comprendido entre Enero de 1997 y Octubre del 2001 (4 años y 10 meses) se realizó la prueba de detección de microfilaremia a 1638 sujetos, de los cuales 30 resultaron positivos para microfilarias de Loa loa.

Se diseñó un estudio de casos y controles. Se definieron como casos los sujetos que procedían de áreas endémicas para filariasis por Loa loa, que consultaron en nuestra Unidad y que presentaban un test de detección de microfilaremia positivo para Loa loa (30 casos). Se seleccionaron tres controles por cada caso de entre los sujetos con microfilaremia negativa a partir de la base de datos del laboratorio de la Unidad (90 controles). La selección se realizó de forma aleatoria y sin ningún criterio de apareamiento.

Las siguientes variables fueron extraídas retrospectivamente de la revisión de las historias clínicas de los sujetos: edad, sexo, país de procedencia, manifestaciones clínicas y presencia de eosinofilia. Las características clínicas y alteraciones analíticas se recogieron coincidiendo en el tiempo con el resultado parasitológico. Se definió inmigrante a aquel individuo natural y procedente de una zona de riesgo y desplazado a nuestro país. Se consideraron como datos clínicos compatibles con el diagnóstico la presencia de edemas de Calabar, alteraciones dermatológicas como prurito o rash y alteraciones oculares como visualización de la filaria, fotofobia, lagrimeo y edema periorbitario. De entre los datos analíticos se recogió la presencia de eosinofília, definida como $\geq 450$ eosinofilos/L en valores absolutos ${ }^{17}$.
Los resultados de la detección de microfilaremia se obtuvieron de la base de datos del laboratorio. El estudio parasitológico de todos los sujetos que se visitan en la Unidad se realiza en el laboratorio de la misma y el diagnóstico de loasis se realiza mediante detección de microfilarias en sangre periférica mediante técnica específica de concentración de las microfilarias por leucoconcentración con saponina. Se recoge una muestra de $3 \mathrm{~mL}$ de sangre periférica durante el día, entre las 11 y las 13 horas, dado el ciclo diurno de la microfilaria de Loa loa, se hemoliza con saponina al $2 \%$ y se estudia al microscopio óptico para detectar microfilarias del nemátodo $^{18}$.

Para la descripción de las variables cualitativas se estimaron proporciones de forma puntual y con intervalos de confianza del 95\%. En la descripción de las variables cuantitativas se estimó la media aritmética y la desviación estándar. Para comparar los dos grupos se utilizó la t de Student para variables cuantitativas, comprobando la normalidad de la distribución y la igualdad de la varianza. En variables cualitativas se utilizó el test de Chi cuadrado, siempre y cuando cumpliera criterios de aplicación (efectivos esperados $\geq 5$ ). Para valorar la asociación se calculó la Odds Ratio de forma puntual y con intervalos de confianza del 95\% según el método de Mantel y Fleiss. Se calcularon los valores predictivos positivos y negativos, la sensibilidad, la especificidad y los falsos negativos para cada una de las alternativas comparadas, tomando como referencia la determinación de microfilaremia a toda la población atendida en la Unidad. Como medida de impacto se estimó el NNS (Number necessary to screen) ${ }^{19-21}$.

\section{RESULTADOS}

\section{Características socio-demográficas, clínicas y analíticas:}

De los 1638 sujetos atendidos a los que se realizó la prueba de microfilaremia, 1,8\% 
$(30 ; 1,2-2,6 \%)$ resultaron positivos. $\mathrm{La}$ media de edad fue de 39,1 años (DS 15,7) en los casos y de 32,2 años (DS 13,4) en los controles $(\mathrm{p}=0,03)$ (tabla 1$)$. En relación al país de procedencia, se detectaron diferencias entre los dos grupos, el 56,7\% (17; 37,4$74,5 \%$ ) de los casos procedían de Guinea Ecuatorial, frente al 28,9\% (26; 9,8-39,4\%) de los controles $(\mathrm{p}=0,006)$. Sin embargo, no se encontraron diferencias estadísticamente significativas entre los casos y los controles respecto al género y a la condición de inmigrante (tabla 1). De los 30 casos con loasis, $76,7 \%(23 ; 57,7-90,1 \%)$ presentaban eosinofilia, siendo ésta casi 9 veces más frecuente en los casos que en los controles (OR 8,8; IC $3,3-23,1 ; \mathrm{p}<0.0001$ ) (tabla 1).

Un 30,0\% (9; 14,7-49,4\%) de los casos presentaba clínica compatible con el diagnóstico, resultando 2,8 veces más frecuente que en los controles (OR 2,8; IC 1,0-7,5; $\mathrm{p}=0,04)$ (tabla 1). En relación a los casos la clínica cutánea (prurito, eritema, rash) era la más frecuente $(8 ; 26,7 \% ; 12,3-45,9 \%)$, encontrándose sólo un caso de visualización de la filaria en la conjuntiva del ojo y ninguno de edema de Calabar. La presencia de clínica y/o eosinofilia fue prácticamente 8 veces superior entre los casos que entre los controles $(\mathrm{OR}=7,9$; IC 2,7-22,7; $\mathrm{p}<0,0001)$ (tabla 1).

\section{Alternativas de cribaje}

Si aplicáramos el test de cribado a toda la población inmigrante procedente de áreas endémicas, deberíamos realizar 54,6 pruebas para detectar un caso (se ha realizado la prueba a 1638 sujetos y en 30 casos la microfilaremia es positiva). Sin embargo, si la

Tabla 1

Comparación de las características sociodemográficas, clínicas y analíticas entre los casos (n=30) y los controles $(n=90)$

\begin{tabular}{|c|c|c|c|c|c|c|c|}
\hline \multirow{2}{*}{ Variables } & \multicolumn{3}{|c|}{ Casos (Loa loa + ) } & \multicolumn{3}{|c|}{ Controles (Loa loa -) } & \multirow[t]{2}{*}{ OR y significación } \\
\hline & $\mathbf{N}$ & $\%$ & IC (\%) & $\mathbf{N}$ & $\%$ & IC \%) & \\
\hline Media de edad & 30 & \multicolumn{2}{|c|}{$\begin{array}{l}39,1 \text { años } \\
\mathrm{DE}=15,7\end{array}$} & 90 & \multicolumn{2}{|c|}{$\begin{array}{l}32,2 \text { años } \\
\mathrm{DE}=13,4\end{array}$} & $\mathrm{p}=0,03$ \\
\hline $\begin{array}{ll}\text { Sexo } & \\
& \begin{array}{l}\text { Hombre } \\
\text { Mujer }\end{array}\end{array}$ & $\begin{array}{l}17 \\
13\end{array}$ & $\begin{array}{l}56,7 \\
43,3\end{array}$ & $\begin{array}{l}37,4-74,5 \\
25,5-62,6\end{array}$ & $\begin{array}{l}60 \\
30\end{array}$ & $\begin{array}{l}65,6 \\
34,4\end{array}$ & $\begin{array}{l}54,8-75,3 \\
24,7-45,2\end{array}$ & $\begin{array}{c}0,7(0,3-1,6) \\
p=0,38\end{array}$ \\
\hline $\begin{array}{r}\text { Inmigración } \\
\text { Sí } \\
\text { No }\end{array}$ & $\begin{array}{c}29 \\
1\end{array}$ & $\begin{array}{c}96,7 \\
3,3\end{array}$ & $\begin{array}{l}82,8-99,9 \\
0,08-17,2\end{array}$ & $\begin{array}{c}84 \\
6\end{array}$ & $\begin{array}{c}93,3 \\
6,7\end{array}$ & $\begin{array}{c}85,9-97,5 \\
2,5-17,1\end{array}$ & $\begin{array}{c}2,1(0,2-18,1) \\
p=0,68\end{array}$ \\
\hline $\begin{array}{r}\text { Eosinofilia }^{1,2} \\
\text { Sí } \\
\text { No }\end{array}$ & $\begin{array}{c}23 \\
7\end{array}$ & $\begin{array}{l}76,7 \\
23,3\end{array}$ & $\begin{array}{c}57,7-90,1 \\
9,9-42,3\end{array}$ & $\begin{array}{l}24 \\
64\end{array}$ & $\begin{array}{l}27,3 \\
72,7\end{array}$ & $\begin{array}{l}18,3-37,8 \\
62,2-81,7\end{array}$ & $\begin{array}{c}8,8(3,3-23,1) \\
\mathrm{p}<0,0001\end{array}$ \\
\hline $\begin{array}{c}\text { Clínica compatible } \\
\text { Sí } \\
\text { No }\end{array}$ & $\begin{array}{c}9 \\
21\end{array}$ & $\begin{array}{l}30,0 \\
70,0\end{array}$ & $\begin{array}{l}14,7-49,4 \\
50,6-85,3\end{array}$ & $\begin{array}{l}12 \\
78\end{array}$ & $\begin{array}{l}13,3 \\
86,7\end{array}$ & $\begin{array}{c}7,1-22,1 \\
77,9-92,9\end{array}$ & $\begin{array}{c}2,8(1,0-7,5) \\
p=0,04\end{array}$ \\
\hline $\begin{array}{r}\text { Clínica } \quad \text { y/o } \\
\text { eosinofilia*, } \dagger \\
\text { Sí } \\
\text { No }\end{array}$ & $\begin{array}{c}25 \\
5\end{array}$ & $\begin{array}{l}83,3 \\
16,7\end{array}$ & $\begin{array}{c}65,3-94,4 \\
5,7-34,7\end{array}$ & $\begin{array}{l}34 \\
54\end{array}$ & $\begin{array}{l}38,6 \\
61,4\end{array}$ & $\begin{array}{l}28,4-49,6 \\
50,4-71,6\end{array}$ & $\begin{array}{c}7,9(2,7-22,7) \\
\mathrm{p}<0,0001\end{array}$ \\
\hline
\end{tabular}

$=450$ eosinófilos $\times 10 / 1$

†Valor de eosinofília desconocido en dos sujetos 
Tabla 2

Sensibilidad (S), especificidad (E), valor predictivo positivo (VP+), valor predictivo negativo (VP-) y número necesario para el cribaje (NNS) para las diferentes alternativas de selección

\begin{tabular}{|l|c|c|c|c|c|}
\hline Criterio de cribaje & $\begin{array}{c}\text { S } \\
(\text { IC) }\end{array}$ & $\begin{array}{c}\text { E } \\
(\text { IC) }\end{array}$ & $\begin{array}{c}\text { VP+ } \\
\text { (IC) }\end{array}$ & $\begin{array}{c}\text { VP- } \\
\text { (IC) }\end{array}$ & $\begin{array}{c}\text { NNS } \\
\text { (IC) }\end{array}$ \\
\hline Clínica & $\begin{array}{c}30,0 \\
(15,4-49,6)\end{array}$ & $\begin{array}{c}86,7 \\
(77,5-92,6)\end{array}$ & $\begin{array}{c}42,9 \\
(22,6-65,6)\end{array}$ & $\begin{array}{c}78,8 \\
(69,2-86,1)\end{array}$ & $\begin{array}{c}40 \\
(23-165)\end{array}$ \\
\hline Eosinofilia1 & $\begin{array}{c}76,7 \\
(57,3-89,4)\end{array}$ & $\begin{array}{c}72,7 \\
(62,0-81,4)\end{array}$ & $\begin{array}{c}48,9 \\
(34,2-63,7)\end{array}$ & $\begin{array}{c}90,1 \\
(80,2-95,6)\end{array}$ & $\begin{array}{c}23 \\
(17-34)\end{array}$ \\
\hline Eosinofilia* y/o clínica & $\begin{array}{c}83,3 \\
(64,5-93,7)\end{array}$ & $\begin{array}{c}61,4 \\
(50,3-71,4)\end{array}$ & $\begin{array}{c}42,4 \\
(29,8-55,9)\end{array}$ & $\begin{array}{c}91,5 \\
(80,6-96,8)\end{array}$ & $\begin{array}{c}29 \\
(21-48)\end{array}$ \\
\hline
\end{tabular}

determinación de microfilaremia se realizara únicamente en los sujetos que presentan eosinofilia y/o clínica compatible, el número de pruebas que deberíamos realizar para cada caso detectado sería inferior $(\mathrm{NNS}=29$; $\mathrm{IC}=21-48)$ pero habría un $16,7 \%(5 ; \mathrm{IC}=5,7$ $34,7 \%$ ) de infectados que no serían diagnosticados (tabla 2).

Si se plantea realizar la determinación de microfilaremia sólo en los sujetos que presentan clínica compatible dejaríamos de diagnosticar a un $70 \%(21 ; 50,6-85,3 \%)$ de los infectados (tabla 2).

\section{DISCUSIÓN}

En los últimos años se ha observado en nuestro entorno un incremento de población inmigrante procedente de países tropicales. En algunas zonas del casco antiguo de la ciudad de Barcelona este incremento es espectacular, el 36,2\% de la población es inmigrante y el $51,0 \%$ de los niños atendidos por los servicios de pediatría son inmigrantes o hijos de inmigrantes ${ }^{22}$. Por ello las patologías propias de los países tropicales han dejado de ser únicamente un problema de las unidades especializadas y se han convertido en una preocupación de los profesionales de atención primaria. Los profesionales se preguntan cómo deben actuar ante estos sujetos. Para ello se han elaborado distintos protoco- los de acogida, basados con frecuencia en la práctica y no tanto en la evidencia, en que se incentiva a los profesionales a realizar pruebas de cribaje para descartar diferentes patologías endémicas en los países de origen ${ }^{16}$.

La aplicación indiscriminada de estos protocolos está comportando un consumo de recursos y unas molestias a los usuarios que no siempre estarían suficientemente justificados. El análisis de la adecuación de cada una de las pruebas que componen dichos protocolos requeriría un abordaje diferente y una evaluación específica. En este sentido el cribaje de la parasitación por Loa loa es un tema históricamente poco debatido. Su detección se incluye en algunos de los protocolos de acogida de población inmigrante procedente de zonas endémicas, aunque esta práctica ha sido poco evaluada.

\section{Características diferenciales sociodemográficas, clínicas y analíticas}

Probablemente las diferencias en la edad que se han observado entre los casos y los controles podrían estar relacionadas con el hecho de que más de la mitad de los casos proceden de Guinea Ecuatorial. La población inmigrante de este origen es de mayor edad que la de otros países endémicos, debido a que el fenómeno migratorio de este país es anterior y de características diferentes. 
La distribución geográfica de loasis no es uniforme en todos los países de Africa central y del oeste de manera que la prevalencia de la misma en la población estudiada está influenciada por el país de origen. Nosotros consideramos válidos los resultados de nuestro estudio debido a las corrientes migratorias en nuestra población.

Sólo una tercera parte de nuestros casos presentaba clínica compatible con el diagnóstico, al igual que en el estudio de Klion ${ }^{10}$. Esto es probablemente debido a que en nuestro estudio prácticamente todos los sujetos incluidos, tanto casos como controles, eran población inmigrante $\mathrm{y}$, tal como se demuestra en estudios previos, la mayor parte de los infectados procedentes de áreas endémicas no presentan clínica compatible con infección por Loa loa. Este estudio confirma de nuevo que la loasis puede pasar con frecuencia desapercibida en población nativa de zonas endémicas, lo que favorece el retraso en el diagnósti$\mathrm{co}^{7,10,11,23}$. Otros autores han encontrado un porcentaje de sujetos asintomáticos inferior, pero el ámbito de estudio es diferente al nuestro ${ }^{11}$. Es importante sospechar esta infección en sujetos asintomáticos o con clínica inespecífica teniendo en cuenta que se trata de una infección con potencial comorbilidad, causada muchas veces por los índices de eosinófilos, y para la cual disponemos de un tratamiento con DEC (dietilcarbamazina), tratamiento efectivo que puede ser monitorizado y controlado en los países receptores de dicha población inmigrante. Diagnosticar la infección por Loa loa a partir de sintomatología patognomónica (edema de Calabar o visualización del gusano adulto en la conjuntiva del ojo) es poco frecuente, de hecho en nuestro estudio sólo detectamos un caso.

En este estudio más de un $75 \%$ de los casos presentaba eosinofilia en el momento de realizar la prueba, al igual que sucedía en otros estudios realizados en población procedente de áreas endémicas ${ }^{10,11}$.

\section{Alternativas de cribado}

En nuestra Unidad, al igual que en algunos centros de atención primaria, se realiza cribaje de infección por Loa loa mediante determinación de microfilaremia a todos los sujetos procedentes de áreas endémicas. Sin embargo, se desconoce si esta es la población en la que el rendimiento de las pruebas de cribado es superior o existe una alternativa mejor.

Nosotros consideramos que la decisión de a qué población debemos realizar las prueba de cribaje debería incorporar el criterio de coste en términos de número de pruebas realizadas para cada caso detectado y el número de casos que dejamos de diagnosticar cuando preseleccionamos una población, es decir el porcentaje de falsos negativos o casos infectados no diagnosticados.

Con relación a cuál es la mejor población en la que realizar el cribaje y teniendo en cuenta los criterios anteriores, todas las alternativas dejan de diagnosticar una proporción considerable de casos infectados, excepto que la prueba se realice a toda la población inmigrante procedente de áreas endémicas. En caso de tomar como criterio de selección para realizar el cribaje la presentación de clínica, un $70 \%$ de la población infectada no sería diagnosticada e incluso filtrando a la población por clínica y/o eosinofilia, una sexta parte de la población enferma tampoco sería detectada.

Por otra parte la probabilidad de que el sujeto que presenta clínica compatible, eosinofilia o la combinación de ambas esté infectado es muy baja, en todos los casos inferior al $50 \%$ pues se trata de signos y síntomas muy inespecíficos, pudiendo estar presentes en un considerable número de patologías de prevalencia superior a la loasis. Sospechamos que la presencia de clínica específica de loasis podría estar infravalorada, ya que fue recogida de forma retrospectiva revisando las historias clínicas. En gene- 
ral, en el momento de realizar la prueba, no había sospecha específica de loasis y por lo tanto la anamnesis no iba dirigida a esta patología.

Con relación a los costes, ninguna de las diferentes alternativas presenta unos costes tan importantes que no puedan ser asumidos por el sistema, si bien existen diferencias en el número de pruebas que deberíamos realizar para diagnosticar un caso. De hecho en la alternativa con un coste superior, determinar la microfilaremia a toda la población inmigrante que provenga de zonas de riesgo, el precio que deberíamos asumir sería de 54,6 pruebas para diagnosticar un caso, relativamente poco alejada del resto de alternativas (40, 29 y 23 pruebas para diagnosticar un caso).

Teniendo en cuenta el coste oportunidad, consideramos que siempre que existan recursos para asumirla y no tengamos una alternativa mejor, la opción más adecuada sería realizar detección de microfilaremia a toda la población inmigrante proveniente de zonas endémicas para loasis. A pesar de ello, si se tuviera que aplicar un criterio de priorización para el cribaje, la población que tiene más probabilidad de estar infectada es la que presenta eosinofilia y/o clínica compatible con el diagnóstico de loasis.

Las conclusiones de estudios como el nuestro, que pretende evaluar la adecuación de realizar una prueba de cribaje, tienen un componente subjetivo importante. En nuestro caso, si la efectividad se hubiera estimado teniendo en cuenta la frecuencia de complicaciones graves de la infección por Loa loa (complicaciones evitadas) y no a partir de las infecciones detectadas, las conclusiones hubieran podido ser diferentes. Sin embargo, es éticamente inadmisible en nuestro entorno dejar de detectar una infección con consecuencias potencialmente graves, aunque infrecuentes, si disponemos de los recursos necesarios. Además, las manifestaciones más comunes de esta infección, aun- que no son graves, son molestas para el sujeto, como por ejemplo el prurito. Las conclusiones también podrían ser muy diferentes en un entorno de escasez de recursos, por lo que las recomendaciones que realizamos no podrían ser directamente extrapolables a otros entornos de baja renta.

A pesar de que nuestra Unidad es centro de referencia para la atención al inmigrante de un amplio territorio el número de casos reclutados ha sido limitado. Resultaría complicado realizar un estudio multicéntrico en la atención primaria de salud, por lo que la única solución razonable para aumentar el número de casos sería incrementar el periodo de reclutamiento.

Nosotros consideramos oportuno realizar este tipo de evaluaciones para otros problemas de salud incluidos en el cribado que se realiza en la población inmigrante, priorizando aquellos en que hay menos evidencias que justifiquen el cribado. También recomendamos que este tipo de evaluación se realice desde el ámbito de la atención primaria, ya que atiende a una población menos seleccionada y por lo tanto más representativa de la población general que la que se atiende en el nivel especializado.

Podemos concluir que estaría indicado practicar un test de cribaje con determinación de microfilarias en sangre periférica a toda la población inmigrante procedente de África Central y del Oeste, áreas hiperendémicas de loasis, independientemente de la presencia de eosinofilia o clínica compatible, siempre que se disponga de los recursos necesarios.

\section{BIBLIOGRAFIA}

1. Sasa M. Human filariasis: a global survey of epidemiology and control. Baltimore: University Park Press; 1976.

2. Goussard B, Ivanoff B, Frost E, Garin Y, Bourderiou C. Age of appearance of IgM, and IgE antibo- 
dies specific for Loa loa in Gabonese children. Microbiol Immunol 1984; 28: 787-92.

3. Farrer WE, Wittner M, Tanowitz HB. African eye worm (Loa loa) in a tourist. Ann Ophthalmol 1981; 13:1177-9.

4. Borrás R, Guna R, Guerrero A, Domínguez MV et al. Diagnostico de las microfilaremias: a propósito de un caso de coparasitación por Loa loa y Mansonella perstans en una mujer ecuatoguineana con miocardiopatía constrictiva e hipereosinofilia periférica. En: Documento de control de calidad. Madrid: Sociedad Española de enfermedades infecciosas y Microbilogía clínica; 2001.

5. Hall CL, Stephens L, Peat D, Chiodini PL. Nephrotic syndrome due to loiasis following a tropical adventure holiday: a case report and review of the literature. Clin Nephrol 2001; 56(3), 247-50.

6. Nutman TB. Case record of the Massachusets general Hospital. NEJM 2002; 346(2): 115-22.

7. Nutman TB, Miller KD, Mulligan M, Ottesen EA. Loa loa infection in temporary residents of endemic regions: recognitions of a hyperresponsive syndrome with characteristic clinical manifestations. J Infect Dis 1986; 154(1):10-8.

8. Nutman TB, Reese W, Poindexter RW, Ottesen EA. Immunologic correlates of the hyperresponsive syndrome of loiasis. J Infect Dis 1988; 157(3): 54450 .

9. Libman MD, MacLean JD, Gyorkos TW. Screening for schistosomiasis, Filariasis and Strongyloidiasis among expatriates returning from the tropics. Cl Infect. Dis. 1993; 17: 353-9.

10. Klion AD, Massougbodji A, Sadeler BC, Ottesen EA, Nutman TB. Loiasis in endemic and nonendemic populations: immunologically mediated differences in clinical presentation. J Infect. Dis 1991; 163:1318-25.

11. Churchill DR, Morris C, Fakoya, Wright SG, Davidson RN. Clinical and Laboratory features of patients with loiasis (Loa loa filariasis) in the U.K. J. Infect 1996; 33: 103-9.

12. Singh B. Molecular methods for diagnosis and epidemiological studies of parasitic infections. Int $\mathrm{J}$ Parasitol 1997; 27(10): 1135-45.
13. Toure FS, Bain O, Nerrienet E, Millet P, Wahl G, Toure Y, Doumbo O, Nicolas L, Georges AJ, McReynolds LA, Egwang TG. Detection of Loa loa-specific DNA in blood from occult-infected individuals. Exp Parasitol 1997; 86: 163-70.

14. Touré FS, Mavoungou E, Deloron P, Egwang TG. Analyse comparative de deux méthodes diagnostiques de la loase humaine: sérologie IgG4 et PCR nichée. Bull Soc Pathol Exot 1999; 92 (3): 167-70.

15. López-Vélez R, Huerga H. La consulta del inmigrante. En: López-Vélez, Huerga, Org. Inmigración y salud. Aproximación desde atención primaria. Madrid: PBMed; 2002. p. 113-24.

16. Camps N, Cardeñosa N, Corachán M, Domínguez A, Faixedas MT, Garrido P, Gómez i Prat, J. Protocol d'actuació per al control de malaties parasitàries en Immigrants. Barcelona: Direcció general de Salut Publica, Generalitat de Catalunya; 2001.

17. Altman PL, Dittmer DS. Blood and other body fluids. Washington DC: Federation of American Societies for experimental biology; 1961.p. 125.

18. Sang H.T, Petitory J. Techniques de concentration des microfilaires sanguicoles. Bull Soc Path Exot 1963; 56:197-206.

19. Cook RJ y Sackett DL. The number needed to treat: a clinically useful measure of treatment effect. BMJ 1995; 310: 452-4.

20. Rembold CM. Number needed to screen: development of a statistic for disease screening. BMJ 1998; 317: 307-12.

21. Sackett DL, Richardson WS, Rosenberg W, Haynes RB. Valoración crítica de la evidencia En: Sackett DL, Richardson WS, Rosenberg W, Haynes RB, org. Medicina basada en la evidencia. Cómo ejercer y enseñar la MBE. Madrid: Momento Médico Iberoamericana; 1997. p. 119-22.

22. Ajuntament de Barcelona. La població estrangera a Barcelona. Disponible en: www.bcn.es/estadistica/catala/dades/inf/est/pobest/index.htm.

23. Rakita R, Clinton A. Loa loa infection as a cause of migratory angioedema: report of three cases from the Texas Medical Center. Clin Infect Dis. 1993; 17: 691-4. 